\title{
Lab on a
}

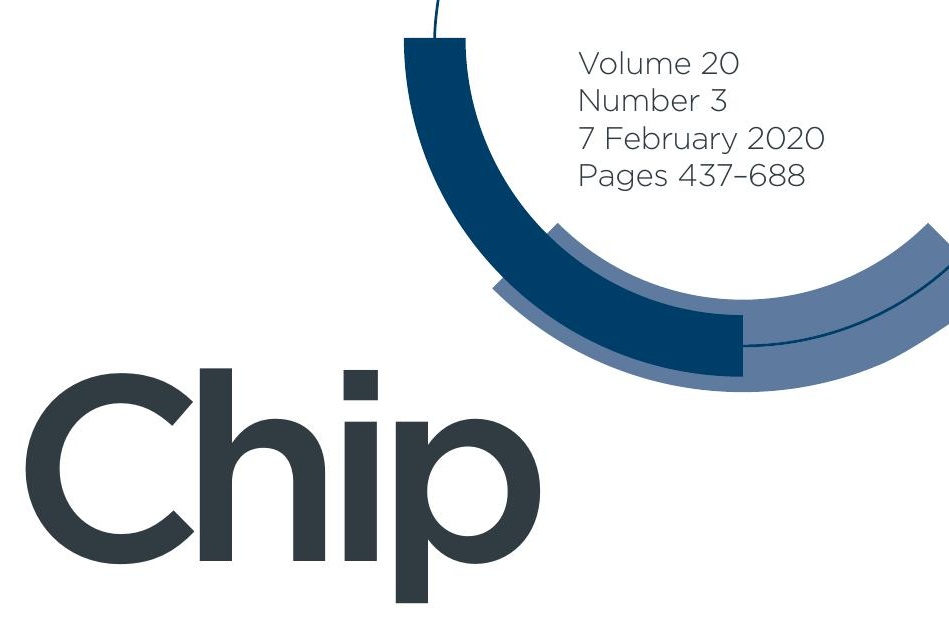

Devices and applications at the micro- and nanoscale rsc.li/loc

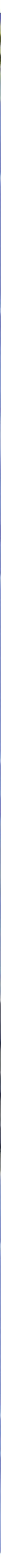

ISSN 1473-0197 


\section{Lab on a Chip}

Check for updates

Cite this: Lab Chip, 2020, 20, 490

Received 1st October 2019,

Accepted 29th November 2019

DOI: 10.1039/c9lc00980a

rsc.li/loc

\section{Engineering 3D parallelized microfluidic droplet generators with equal flow profiles by computational fluid dynamics and stereolithographic printing $\dagger$}

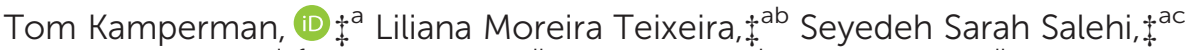 \\ Greet Kerckhofs, ${ }^{\text {defg }}$ Yann Guyot, ${ }^{\text {dh }}$ Mike Geven,' Liesbet Geris, ${ }^{\text {dh }}$ Dirk Grijpma, \\ Sebastien Blanquer ${ }^{j}$ and Jeroen Leijten (iD *a
}

\begin{abstract}
Microfluidic droplet generators excel in generating monodisperse micrometer-sized droplets and particles. However, the low throughput of conventional droplet generators hinders their clinical and industrial translation. Current approaches to parallelize microdevices are challenged by the two-dimensional nature of the standard fabrication methods. Here, we report the facile production of three-dimensionally (3D) parallelized microfluidic droplet generators consisting of stacked and radially multiplexed channel designs. Computational fluid dynamics simulations form the design basis for a microflow distributor that ensures similar flow rates through all droplet generators. Stereolithography is the selected technique to fabricate microdevices, which enables the manufacturing of hollow channels with dimensions as small as $50 \mu \mathrm{m}$. The microdevices could be operated up to 4 bars without structural damage, including deformation of channels, or leakage of the on-chip printed Luer-Lok type connectors. The printed microdevices readily enable the production of water-in-oil emulsions, as well as polymer containing droplets that act as templates for both solid and core-shell hydrogel microparticles. The cytocompatibility of the 3D printed device is demonstrated by encapsulating mesenchymal stem cells in hydrogel microcapsules, which results in the controllable formation of stem cell spheroids that remain viable and metabolically active for at least 21 days. Thus, the unique features of stereolithography fabricated microfluidic devices allow for the parallelization of droplet generators in a simple yet effective manner by enabling the realization of (complex) 3D designs.
\end{abstract}

\section{Introduction}

Microfluidic generation of droplets provides precise volume control, low reagent consumption, in-line control, and monodisperse products. ${ }^{1-4}$ Not surprisingly, microfluidics is highly suitable for numerous applications including (single) cell analysis, cell therapies, pharmacological screenings,

\footnotetext{
${ }^{a}$ Department of Developmental BioEngineering, Faculty of Science and Technology, Technical Medical Centre, University of Twente, Drienerlolaan 5, 7522 NB Enschede, The Netherlands. E-mail: jeroen.leijten@utwente.nl

${ }^{b}$ Regenerative Medicine Utrecht, Department of Equine Sciences, Faculty of Veterinary Medicine, Utrecht University, Utrecht, The Netherlands

${ }^{c}$ Department of Mechanical Engineering, Sharif University of Technology, P.O. Box: 11155-9567, Tehran, Iran

${ }^{d}$ Prometheus, Division of Skeletal Tissue Engineering, KU Leuven, Herestraat 49, 3000 Leuven, Belgium

${ }^{e}$ Department Materials Engineering, KU Leuven, Kasteelpark Arenberg 44, 3001 LEUVEN, Belgium

${ }^{f}$ Biomechanics Lab - Institute of Mechanics, Materials, and Civil Engineering, UCLouvain, Place du Levant 2/L5.04.02, 1348, Louvain-la-Neuve, Belgium
}

emulsion chemistry, and micromaterial fabrication. ${ }^{5-9}$ However, several practical problems that occur during microfluidic device parallelization have been hampering the upscaling of microfluidic processes to clinically and industrially relevant quantities. Specifically, upscaling is currently limited by the two dimensional (2D) or 2.5D nature of conventional microfluidic device fabrication methods,

\footnotetext{
${ }^{g}$ IREC - Institut de Recherche Expérimentale et Clinique, UCLouvain, Avenue Hippocrate, 55 bte B1.55.02, 1200 Woluwé-Saint-Lambert, Belgium

${ }^{h}$ Biomechanics Research Unit, GIGA in silico medicine, Université de Liège, Avenue de l'Hopital 11, 4000 Liège, Belgium

${ }^{i}$ Department of Biomaterials Science and Technology, Faculty of Science and Technology, Technical Medical Centre, University of Twente, Drienerlolaan 5, 7522NB Enschede, The Netherlands

${ }^{j}$ Institut Charles Gerhardt Montpellier - UMR5253, Université Montpellier, CNRS, ENSCM, Montpellier, France

$\dagger$ Electronic supplementary information (ESI) available: Experimental section; Fig. S1-S6. See DOI: 10.1039/c9lc00980a

$\$$ Co-first authors who contributed equally to this work. The author sequence was randomly determined.
} 
which only provide parallelization along single- or double-axis planes. ${ }^{10-12}$ Although additive manufacturing technologies such as extrusion printing, two photon polymerization, and stereolithography have been used to fabricate three dimensional (3D) microfluidic devices, ${ }^{13-20}$ the multi-axial stacking of high-resolution microfluidic droplet generators along more than two axes remains elusive. Moreover, parallelization of microfluidic droplet generators in current designs is typically associated with broadened droplet size distributions due to the small variances in flow rates that typically occur across multiplexed devices. ${ }^{21,22}$ Current parallelization strategies thus often fail to preserve one of microfluidics' key advantages, that is, monodisperse production. Various liquid distribution schemes including 'tree', 'ladder', and 'annular' type channel networks have been used to minimize the pressure gradients across parallelized droplet generators. ${ }^{10,23-26}$ However, the relatively complex designs of these liquid distributors have hampered the controlled parallelization along more than two axes. To overcome this limitation and enable the large-scale generation of monodisperse microdroplets, novel advanced designing and manufacturing strategies are needed to achieve the high-resolution fabrication of 3D parallelized microfluidic device designs with equally distributed flow profiles.

In this work, we used computational fluid dynamics simulations in combination with high-resolution stereolithographic printing to engineer 3D parallelized microfluidic droplet generators. The devices had a minimal footprint and were compatible with high-throughput production of monodisperse micromaterials. Specifically, multiple stacks of radially oriented droplet generators were connected to a microflow distributor that was optimized to provide equal flows to all connected channels. This computed microfluidic device design thereby enabled the continuous high-throughput generation of monodisperse droplets using multiple 3D stacked droplet generators operating at equal flow rates. The microfluidic devices were fabricated in a cleanroom-free manner that did not demand labor intensive post-processing or assembly. Specifically, we used stereolithography with a digital micromirror device (DMD), also called digital light processing (DLP), to create optically transparent flow focusing droplet generators with channel diameters as small as $50 \mu \mathrm{m}$ with high fidelity. The 3D parallelized microfluidic device was demonstrated to be compatible with the production of microdroplets and particles of various sizes, compositions, and complexities. For example, human mesenchymal stem cells were encapsulated into hydrogel microcapsules that acted as controlled microbioreactors, and which can potentially be applied in fundamental biological research, pharmaceutical screening, or therapeutic applications. ${ }^{5}$ In short, the combination of computational fluid dynamics simulations and stereolithographic printing enabled the engineering of a 3D parallelized microfluidic device with a minimal footprint and compatible with high-throughput production of monodisperse micromaterials. This microfluidic upscaling strategy overcomes key hurdles that are associated with conventional droplet generator parallelization strategies in a facile, affordable, and easily adoptable manner.

\section{Results and discussion}

\section{Stereolithographic 3D printing of micrometer-sized channels}

Stereolithographic printing using a DMD is compatible with the facile and reproducible printing of optically transparent devices with intricate internal features, such as $3 \mathrm{D}$ microfluidic channel networks. ${ }^{27,28}$ Importantly, the potential of $3 \mathrm{D}$ microfluidic device printing is mostly limited by the minimal printing resolution of continuous void spaces (e.g., open channels). Besides the DMD mirror size, dominant factors affecting the printing resolution are the spectral overlap of the light source and the resin's light absorber, as well as the absorber concentration and irradiation dose. ${ }^{17} \mathrm{We}$ explored a visually transparent, yet photocurable orange resin (PIC100) that allowed for high-fidelity 3D photo-based printing of optically transparent microfluidic chips. This commercially available resin in combination with the used printer (EnvisionTec Perfactory 3 Mini Multi Lens) readily allowed for the fabrication of open and transparent microfluidic channels with dimensions down to $50 \mu \mathrm{m}$ using the pre-optimized exposure time and intensity of $9 \mathrm{~s}$ and 180 $\mathrm{mW} \mathrm{dm}{ }^{-2}$, respectively (Fig. $1 \mathrm{a}$ and b). ${ }^{29}$ Deviating from these

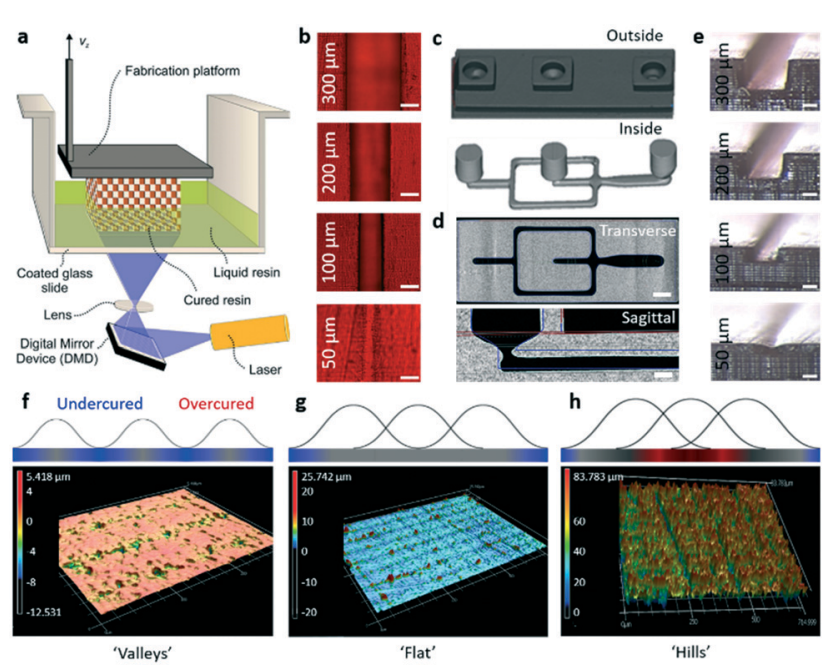

Fig. 1 High-resolution stereolithographic 3D printing of microfluidic channels. (a) Schematic representation of stereolithography with a digital micromirror device (DMD), which was used to manufacture (b) microfluidic channels made of PIC100 resin. Scale bars indicate 100 $\mu \mathrm{m}$. The designed (i.e., intended) channel widths are indicated per microphotograph with white text. (c) 3D reconstructed $\mu \mathrm{CT}$ data of the printed microfluidic device. (d) Cross-sectional $\mu \mathrm{CT}$ images were digitally overlapped with the original CAD design (blue lines) and confirmed the high 3D print fidelity. Scale bar transverse plane indicates $500 \mu \mathrm{m}$. Scale bar sagittal plane indicates $150 \mu \mathrm{m}$. (e) Stereomicroscopic images of open-top channels confirmed accurate inter-layer alignment. Scale bar indicates $300 \mu \mathrm{m}$. (f-h) Laser-scanned surface roughness of the 3D printed channels as determined by confocal profilometry. Voxel undercuring and overcuring resulted in apparent 'valleys' and 'hills' at the voxels' intersection, respectively. 
optimal settings could cause significant overexposure resulting in overlapping crosslinking regions, which caused closure of the smallest channels (Fig. S1 $\dagger$ ). For custom resin formulations and printers, it is therefore crucial to first optimize the level of photocuring in order to optimize the fabrication resolution. To this end, a 'working curve' that correlates the curing depth to the irradiation dose can be constructed. $^{30}$

Computer-aided design (CAD) software and stereolithographic printing of PIC100 resin were then used to manufacture a flow focusing droplet generator with a $100 \mu \mathrm{m}$ wide nozzle. 3D reconstructed microcomputed tomography $(\mu \mathrm{CT})$ analysis confirmed the smooth, continuous, and connected nature of microscale channels throughout the device (Fig. 1c). By digitally overlapping the $\mu \mathrm{CT}$ data with the corresponding CAD design, it was confirmed that the stereolithographic printing of microfluidic channels was associated with a high degree of fidelity as only micrometersized discrepancies were detected (Fig. 1d). Interestingly, these discrepancies predominantly occurred in the smallest channels, which were designed to be $100 \mu \mathrm{m}$ high, but were consistently printed as $75 \mu \mathrm{m}$ high channels with well-defined edges. This phenomenon could potentially be leveraged to controllably achieve 3D printing of hollow channels shallower than $100 \mu \mathrm{m}$. Printing channels with dimensions below 100 $\mu \mathrm{m}$ was also associated with channel rounding. It is anticipated that even smaller channels can be printed when using customized resins, combinations of multiple resins within a single print, and higher resolution 3D printers. ${ }^{17,31}$

An open-top droplet generator was then printed to facilitate characterization of the inter-layer alignment, as well as the printed channels' surface roughness. Importantly, small vibrations of the work bench during printing could introduce notable inter-layer displacements (Fig. S2†). Placing the printer on an isolated work bench was critical to achieve an inter-layer horizontal alignment error of $2.8 \pm 2.8$ $\mu \mathrm{m}$ (average \pm standard deviation; $n=35$ ), as confirmed by cross-sectional microscopic analysis (Fig. 1e). Laser confocal scanning microscopy was used to generate a partial surface profile map, which revealed that the average surface roughness of the channels was as low as $715 \pm 12 \mathrm{~nm}$. Spatial reconstruction of the surface revealed a grid-like pattern that was likely caused by partial overlapping of the projected light from adjacent digital micromirrors (Fig. 1f-h). Consequently, tuning the exposure at the projected intersections affected the channel surface smoothness. Specifically, under- and overexposing individual voxels resulted in the formation of apparent hills or valleys at the voxels' intersections. The relatively rough surfaces that appeared after overcuring were associated with aqueous channel wetting, which led to uncontrolled droplet pinch-off and thus more polydisperse water-in-oil droplet size distributions (Fig. S3†). However,

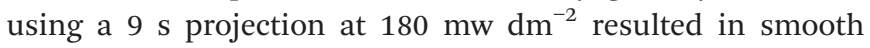
and flat microfluidic channels that enabled the flow focusing of water in oil without wetting of the channel's surface by the aqueous phase.

\section{D printing microfluidic droplet generators}

The optimized stereolithographic 3D printing procedure was used to produce a microfluidic droplet generator (Fig. 2a). The printed microdevices were ready for use after removal of the non-crosslinked resin, which was achieved by flushing the channels with isopropanol. The combination of CAD and 3D printing offered the opportunity to manufacture on-chip integrated Luer-Lok type connectors enabling swift and robust connection of tubing (Fig. 2b). The 3D printed microfluidic channels did not significantly deform with operating pressures up to 4 bar. In contrast, PDMS-based microfluidic devices with identical channel design elastically deformed up to $20 \%$ when exposed to similar pressures, which has been reported to affect the flow velocity, increase the response time, and induce the risk of PDMS/glass delamination resulting in device leakage (Fig. 2c). ${ }^{32}$

The functionality of the 3D printed droplet generators was demonstrated by producing a fluorescently labeled water-inoil emulsion. Specifically, a fluorescein solution was coflowed with Span 80 containing hexadecane, which effectively created stable aqueous microdroplets (Fig. 2d) that could be collected off-chip (Fig. 2e). Of note, this experiment also confirmed that the $3 \mathrm{D}$ printed microdevices were sufficiently transparent to enable on-chip monitoring using conventional optical imaging techniques, such as light microscopy. Monodisperse droplets (coefficient of variation CV $<10 \%$ ) between $150 \mu \mathrm{m}$ and $1000 \mu \mathrm{m}$ in diameter were produced
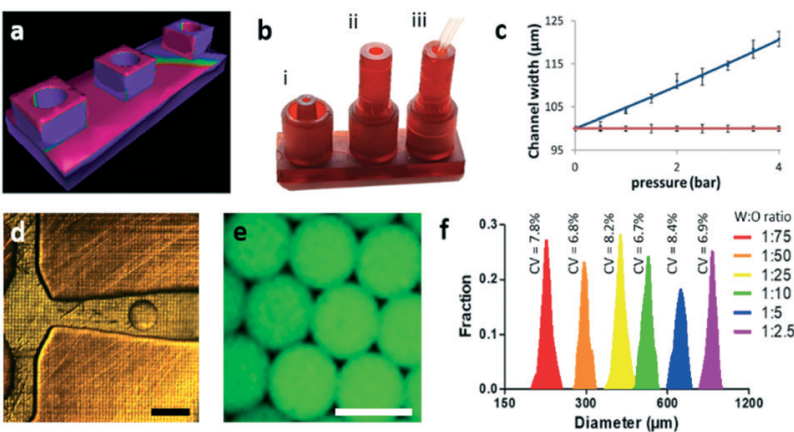

Fig. 2 Microdroplet production using a 3D printed microfluidic device. (a) Stereolithographic printing enabled the printing of a microfluidic droplet generator that could be readily provided with (b) Luer-Lok type connectors, which enable swift chip-to-world connections as visualized by a (i) male Luer-Lok connector, (ii) that can be locked to a separately printed female Luer-Lok, (iii) through which microfluidic tubing can be connected. (c) Channel width (average \pm standard deviation; $n=3$ ) analysis as a function of internal pressure revealed no measurable pressure-induced deformation of 3D printed channels (red curve) in contrast to conventional PDMS-on-glass channels (blue curve) with identical designs. (d) Microphotograph of water-in-oil droplet generation using a 3D printed microfluidic device with a $150 \mu \mathrm{m}$ wide nozzle confirmed chip functionality as well as the transparent nature of the photocured PIC100 resin allowing for on-chip monitoring using light microscopy. (e) Emulsified aqueous droplets containing fluorescein could be collected and imaged off-chip. (f) Size distribution and coefficient of variation (CV) of microdroplets ( $n \geq 100$ ) produced using the 3D printed microfluidic device using various water/oil flow ratios (total flow rate $200 \mu \mathrm{l} \mathrm{min}^{-1}$ ). All scale bars indicate $200 \mu \mathrm{m}$ 
using a $150 \mu \mathrm{m}$ flow focusing droplet generator by varying the per-nozzle water/oil flow ratio from $1: 75$ to $1: 2.5 \mu \mathrm{l}$ $\min ^{-1}$ and maintaining a total per-nozzle flow rate of $200 \mu \mathrm{l}$ $\min ^{-1}$ (Fig. 2f). Operating the droplet generator at significantly higher flow rates causes the transition from the dripping regime to the jetting regime, which results in the formation of more polydisperse droplets, as described in previous studies. ${ }^{33,34}$

\section{D parallelization of microfluidic droplet generators}

Stereolithographic 3D printing allowed for the manufacturing of droplet generator arrays. To demonstrate this, a 4 by 2 array of microfluidic droplet generators was printed within 20 minutes (Fig. S4†). The number of microfluidic droplet generators per array can easily be further upscaled within the same manufacturing time as it is only limited by the printing platform size. Importantly, the accurate and precise nature of stereolithographic 3D printing offers the advantage of manufacturing microfluidic devices with truly complex multilayered channel designs in a facile manner. ${ }^{35}$ To demonstrate this feat, three stacked microfluidic droplet generators with a minimal channel geometry of $75 \mu \mathrm{m}$ were printed. Connecting these stacked droplet generators via a nonoptimized (i.e., conventional) distribution channel resulted in unequal flow distributions across the parallelized device, as

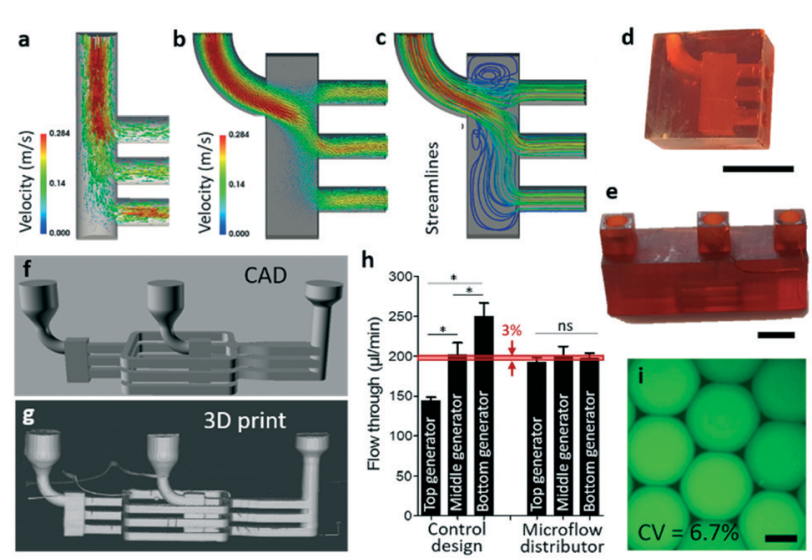

Fig. 3 Design and optimization of the 3D printed stacked microfluidic droplet generator. (a) Numerical flow simulations of conventionally stacked microfluidic channels and (b and c) stacked channels connected via a computationally optimized microflow distributor. (d) Photographs of a 3D printed optimized microflow distributor and (e) a one-piece 3D printed microfluidic device containing microflow distributors serially connected to three stacked droplet generators. Scale bars indicate $500 \mu \mathrm{m}$. Printing fidelity was investigated by comparing the (f) CAD design to $(g)$ 3D reconstructed $\mu C T$ data of the printed microdevice. (h) The measured flow throughs (average \pm standard deviation; $n=3$ ) of conventionally stacked droplet generators (i.e., control design) and stacked droplet generators connected via the computationally designed microflow distributor. ' $*$ ' indicates $p<0.05$; 'ns' indicates $p>0.05$; one-way ANOVA. (i) Microphotograph of monodisperse ( $C V<10 \% ; n \geq 100$ ) fluorescein containing aqueous microdroplets in oil that were produced using stacked microfluidic droplet generators containing a microflow distributor. Scale bar indicates $100 \mu \mathrm{m}$. visualized through computational fluid dynamics simulations (Fig. 3a). Such flow rate gradients across parallelized droplet generators have been reported to cause polydisperse droplet size distributions. ${ }^{21}$ To achieve stacked droplet generators with equal in-channel fluid velocities and flow stream lines, we designed and computationally optimized a microflow distribution tank (Fig. 3b and c). The design was iterated until the relative difference between flow throughs of the three outlets was smaller than $3 \%$, which in principle corresponds to a relative droplet diameter difference of maximally $\sim 1 \%$ (diameter $\sim$ volume ${ }^{1 / 3}$ ). Scanned design parameters included the chamber size, presence of an internal spacer within the chamber, and placement and configuration of chamber inlets and outlets (Fig. S5†). The design iterations eventually resulted in a microflow distributor that was characterized by equally distributed flow rates through all the stacked outlet channels.

The optimized microflow distributor was converted into a CAD model and could be successfully printed as a standalone unit (Fig. 3d), as well as in series with a microfluidic droplet generator stack (Fig. 3e). HCT scanning confirmed that the device - including its internal microchannels - could be $3 \mathrm{D}$ printed with high fidelity as compared to its CAD design (Fig. 3f and g). Measuring the per-channel flow rate confirmed that optimizing the microflow distributor's design reduced the average relative flow rate differences among stacked droplet generators from $>40 \%$ (i.e., not optimized) to $<3 \%$ (i.e., optimized) (Fig. 3h). Consequently, the computed microflow distributor enabled the parallelized production of monodisperse microdroplets enabling equal performance of stacked droplet generators (Fig. 3i).

To achieve 3D parallelization (i.e., along three axes) of the microfluidic device, stacked microfluidic droplet generators were arranged in a radial manner (Fig. 4a and S6†). It is of note that, in contrast to conventional microfabrication methods, stereolithographic printing enabled manufacturing
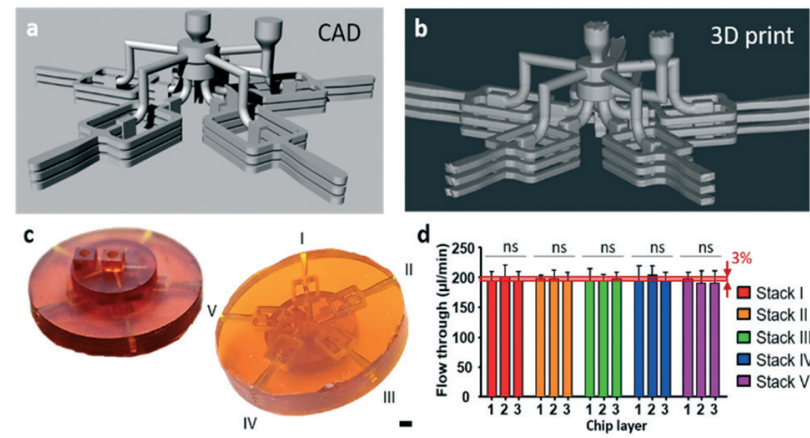

Fig. 4 Printed 3D parallelized microfluidic device containing radially organized microdroplet generator stacks. (a) CAD design and (b) 3D reconstructed $\mu \mathrm{CT}$ data of the printed 3D parallelized microfluidic droplet generator device containing five radially organized stacks that each contain three droplet generators. (c) Photographs of a printed 3D parallelized microfluidic device from the top and bottom views. (d) The measured flow throughs (average \pm standard deviation; $n=3$ ) of fifteen 3D parallelized droplet generators. ' $n s$ ' indicates $p>0.05$; oneway ANOVA. Scale bar indicates $200 \mu \mathrm{m}$ 
of these complex 3D parallelized microdevices at a similar level of ease to the single droplet generator microdevices. ${ }^{13}$ $\mu \mathrm{CT}$ analysis confirmed microchannel connectivity and smoothness of all 3D parallelized droplet generators (Fig. 4b). The flow rates throughout the 3D parallelized droplet generators were not significantly different and fell within the predicted 3\% error (Fig. 4c and d). Advantageously, the upscaling of microdroplet generation throughputs using radial and stacked designs is associated with improved per-footprint production rates. It is of note that the number of droplet generators within the stacked radial microfluidic devices is not limited to their current three by five parallelized design, but can be further increased with the $3 \mathrm{D}$ printer's maximal production dimensions being the key limiting factor.

\section{Cell-laden microgel production using 3D parallelized microfluidic droplet generators}

The 3D parallelized microfluidic droplet generators were compatible with the production of micromaterials in a highly controlled and cytocompatible manner. To demonstrate this, a solution of tyramine-conjugated dextran (Dex-TA) polymer and horseradish peroxidase (HRP) was emulsified in hexadecane oil with Span 80 surfactant. Diffusion-based supplementation of hydrogen peroxide resulted in dextran hydrogel formation through the HRP-mediated crosslinking of tyramines bonds (Fig. 5a). ${ }^{36}$ By tuning the hydrogen peroxide concentration, both solid microgels and hollow hydrogel microcapsules could be fabricated in a controlled

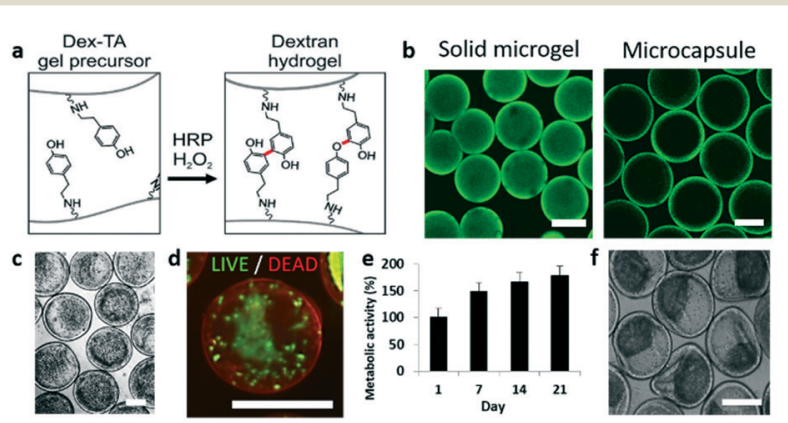

Fig. 5 Production of cell-laden enzymatically crosslinked Dex-TA hollow microgels using a printed 3D parallelized microfluidic device. (a) Schematic representation of enzymatic crosslinking of the Dex-TA polymer using horseradish peroxidase (HRP) and hydrogen peroxide $\left(\mathrm{H}_{2} \mathrm{O}_{2}\right)$. On-chip emulsification followed by off-chip crosslinking based on outside-in diffusion of $\mathrm{H}_{2} \mathrm{O}_{2}$ enabled the formation of (b) solid microgels and hollow microcapsules, as confirmed by confocal microscopy of FITC labeled Dex-TA. (c) Human mesenchymal stem cells (MSC) were encapsulated in Dex-TA microcapsules. Short- and long-term cytocompatibility of the microencapsulation procedure as well as the Dex-TA microcapsules was confirmed by (d) viability analysis (i.e., live/dead) immediately after encapsulation and (e) metabolic activity analysis (i.e., MTT; average \pm standard deviation; $n=$ 3) over a period of 21 days. (f) Furthermore, microencapsulated MSCs remained viable and functional as confirmed by the formation of $3 D$ cellular spheroids within Dex-TA microcapsules. All scale bars indicate $200 \mu \mathrm{m}$. manner (Fig. 5b). Hydrogel microcapsules represent a 3D physically confined microenvironment that is highly suited for the generation of cellular spheroids and organoids, which have a wide variety of applications in biomedical research. ${ }^{37}$ As a proof of concept, mesenchymal stem cells (MSCs) were encapsulated into monodisperse dextran microcapsules with a diameter and shell thickness of $\sim 325 \mu \mathrm{m}$ and $\sim 25 \mu \mathrm{m}$, respectively (Fig. 5c). The high percentage of viable cells (>95\%) post-encapsulation confirmed the cytocompatible nature of cell encapsulation using the printed microfluidic device (Fig. 5d). Furthermore, MSCs encapsulated within the Dex-TA microcapsules remained viable and metabolically active over a culture period of three weeks (Fig. 5e). Due to the bio-inert nature of the hollow microgels' Dex-TA polymer shell, MSCs energetically favored cell-cell interactions over cell-substrate interactions resulting in the efficient formation of stem cell spheroids within the microgels (Fig. 5f). Furthermore, the cell-laden Dex-TA microcapsules remained intact during three weeks of in vitro culture. The high production rate of the printed $3 \mathrm{D}$ parallelized microdevice and its compatibility with biomedical applications (e.g., longterm 3D stem cell culture within hydrogel microcapsules) offers novel opportunities in the fields of developmental biology, drug screening, and bottom-up tissue engineering.

\section{Conclusions}

Upscaling droplet microfluidics through parallelization is technically challenging due to the design complexity of the microfluidic device that is essential to maintain monodisperse production. In this work, we have pioneered a combinatorial approach of computational fluid dynamics simulations, $\mathrm{CAD}$, and $3 \mathrm{D}$ printing to engineer a microflow distribution channel that enabled the 3D parallelization of microfluidic droplet generators while maintaining this desired monodisperse production. Specifically, a commercially available stereolithographic printer equipped with a DMD was leveraged to manufacture microfluidic devices with channel dimensions down to $50 \mu \mathrm{m}$. The $3 \mathrm{D}$ parallelized microfluidic device comprised radially oriented droplet generator stacks containing a computationally designed microflow distributor. This novel 3D parallelization strategy enabled equal per-nozzle flow rates resulting in the production of monodisperse $(\mathrm{CV}<10 \%)$ microdroplets with diameters between 150 and $1000 \mu \mathrm{m}$ by tuning the water/oil flow ratio. Moreover, droplets and micromaterials of different compositions and complexities were produced, including solid microgels and hydrogel microcapsules. To demonstrate the compatibility of the printed microdevice with biomedical applications, viable and long-term stable 3D stem cell spheroids were formed and cultured within dextran-based hydrogel microcapsules. In principle, 3D parallelization could be further upscaled in a facile manner by increasing the number of radial stacks or increasing the amount of droplet generators per stack while maintaining a small production device footprint. It is of note that the resolution of our 
stereolithographic printing method to create continuous open microfluidic channels was limited to $50 \mu \mathrm{m}$. This challenged the generation of $<100 \mu \mathrm{m}$ droplets. However, it is anticipated that higher resolution channel printing can be achieved by improving the printing techniques and exploring novel inks/resins. In short, stereolithographic printing of $3 \mathrm{D}$ parallelized microfluidic droplet generators has significant potential to simplify and accelerate micromaterial production for biomedical, life sciences, cosmetics, food, and pharmacological applications.

\section{Conflicts of interest}

There are no conflicts of interest to declare.

\section{Acknowledgements}

We would like to thank Gabriel Probst (Dept. Mechanical Engineering, KU Leuven) for the expert assistance on overlapping $\mathrm{CAD}$ and $\mu \mathrm{CT}$ data. The X-ray computed tomography images have been generated on the X-ray computed tomography facilities of the Department Materials Engineering of the KU Leuven, financed by the Hercules Foundation (project AKUL 09/001: micro- and nano-CT for the hierarchical analysis of materials). J. L. acknowledges financial support from an Innovative Research Incentives Scheme Vidi award (\#17522) from the Netherlands Organization for Scientific Research (NWO), the European Research Council (ERC, Starting Grant, \#759425), and the Dutch Arthritis Society (\#17-1-405).

\section{References}

1 R. Seemann, M. Brinkmann, T. Pfohl and S. Herminghaus, Rep. Prog. Phys., 2012, 75, 016601.

2 T. Thorsen, R. W. Roberts, F. H. Arnold and S. R. Quake, Phys. Rev. Lett., 2001, 86, 4163-4166.

3 S. L. Anna, N. Bontoux and H. A. Stone, Appl. Phys. Lett., 2003, 82, 364-366.

4 D. R. Link, S. L. Anna, D. A. Weitz and H. A. Stone, Phys. Rev. Lett., 2004, 92, 054503.

5 T. Kamperman, M. Karperien, S. Le Gac and J. Leijten, Trends Biotechnol., 2018, 36, 850-865.

6 C. M. Ho, S. H. Ng, K. H. Li and Y. J. Yoon, Lab Chip, 2015, 15, 3627-3637.

7 G. M. Whitesides, Nature, 2006, 442, 368-373.

8 S. Xu, Z. Nie, M. Seo, P. Lewis, E. Kumacheva, H. A. Stone, P. Garstecki, D. B. Weibel, I. Gitlin and G. M. Whitesides, Angew. Chem., Int. Ed., 2005, 44, 724-728.

9 A. S. Mao, J. W. Shin, S. Utech, H. Wang, O. Uzun, W. Li, M. Cooper, Y. Hu, L. Zhang, D. A. Weitz and D. J. Mooney, Nat. Mater., 2017, 16, 236-243.

10 T. Nisisako, T. Ando and T. Hatsuzawa, Lab Chip, 2012, 12, 3426-3435.

11 D. M. Headen, J. R. García and A. J. García, Microsyst. Nanoeng., 2018, 4, 17076.
12 D. Bardin, M. R. Kendall, P. A. Dayton and A. P. Lee, Biomicrofluidics, 2013, 7, 34112.

13 S. Waheed, J. M. Cabot, N. P. Macdonald, T. Lewis, R. M. Guijt, B. Paull and M. C. Breadmore, Lab Chip, 2016, 16, 1993-2013.

14 R. Song, M. S. Abbasi and J. Lee, Microfluid. Nanofluid, 2019, 23, 92.

15 J. M. Zhang, E. Q. Li, A. A. Aguirre-Pablo and S. T. Thoroddsen, RSC Adv., 2016, 6, 2793-2799.

16 J. M. Zhang, A. A. Aguirre-Pablo, E. Q. Li, U. Buttner and S. T. Thoroddsen, RSC Adv., 2016, 6, 81120-81129.

17 H. Gong, B. P. Bickham, A. T. Woolley and G. P. Nordin, Lab Chip, 2017, 17, 2899-2909.

18 T. Femmer, A. Jans, R. Eswein, N. Anwar, M. Moeller, M. Wessling and A. J. Kuehne, ACS Appl. Mater. Interfaces, 2015, 7, 12635-12638.

19 A. K. Au, W. Huynh, L. F. Horowitz and A. Folch, Angew. Chem., Int. Ed., 2016, 55, 3862-3881.

20 Q. Ji, J. M. Zhang, Y. Liu, X. Li, P. Lv, D. Jin and H. Duan, Sci. Rep., 2018, 8, 4791.

21 E. Amstad, X. Chen, M. Eggersdorfer, N. Cohen, T. E. Kodger, C. L. Ren and D. A. Weitz, Phys. Rev. E, 2017, 95, 043105 .

22 S.-H. Kim, J. W. Kim, D.-H. Kim, S.-H. Han and D. A. Weitz, Microfluid. Nanofluid., 2013, 14, 509-514.

23 D. Conchouso, D. Castro, S. A. Khan and I. G. Foulds, Lab Chip, 2014, 14, 3011-3020.

24 M. B. Romanowsky, A. R. Abate, A. Rotem, C. Holtze and D. A. Weitz, Lab Chip, 2012, 12, 802-807.

25 M. Muluneh and D. Issadore, Lab Chip, 2013, 13, 4750-4754.

26 W. Li, J. Greener, D. Voicu and E. Kumacheva, Lab Chip, 2009, 9, 2715-2721.

27 A. I. Shallan, P. Smejkal, M. Corban, R. M. Guijt and M. C. Breadmore, Anal. Chem., 2014, 86, 3124-3130.

28 A. K. Au, W. Lee and A. Folch, Lab Chip, 2014, 14, 1294-1301.

29 M. A. Geven, V. Varjas, L. Kamer, X. Wang, J. Peng, D. Eglin and D. W. Grijpma, Polym. Adv. Technol., 2015, 26, 1433-1438.

30 F. P. Melchels, J. Feijen and D. W. Grijpma, Biomaterials, 2009, 30, 3801-3809.

31 H. Gong, M. Beauchamp, S. Perry, A. T. Woolley and G. P. Nordin, RSC Adv., 2015, 5, 106621-106632.

32 C. Roh, J. Lee and C. Kang, Molecules, 2016, 21, 798.

33 A. S. Utada, A. Fernandez-Nieves, H. A. Stone and D. A. Weitz, Phys. Rev. Lett., 2007, 99, 094502.

34 T. Kamperman, B. van Loo, M. Gurian, S. Henke, M. Karperien and J. Leijten, Lab Chip, 2019, 19, 1977-1984.

35 S. B. G. Blanquer, M. Werner, M. Hannula, S. Sharifi, G. P. R. Lajoinie, D. Eglin, J. Hyttinen, A. A. Poot and D. W. Grijpma, Biofabrication, 2017, 9, 025001.

36 T. Kamperman, S. Henke, C. W. Visser, M. Karperien and J. Leijten, Small, 2017, 13, 1603711.

37 X. Yin, B. E. Mead, H. Safaee, R. Langer, J. M. Karp and O. Levy, Cell Stem Cell, 2016, 18, 25-38. 